Advances in Geosciences, 2, 173-176, 2005

SRef-ID: 1680-7359/adgeo/2005-2-173

European Geosciences Union

(c) 2005 Author(s). This work is licensed

under a Creative Commons License.

\title{
Nowcasting of convective cells over Italian Peninsula
}

\author{
C. M. Medaglia, C. Adamo, M. Formenton, and F. Piccolo \\ Istituto di Scienze dell'Atmosfera e del Clima (ISAC-CNR Roma), Via Fosso del Cavaliere 100, 00133 Roma, Italy
}

Received: 10 December 2004 - Revised: 18 April 2005 - Accepted: 18 April 2005 - Published: 30 May 2005

\begin{abstract}
The aim of the study is the individuation of convective cells over the Italian peninsula with the conjunction use of geostationary satellite data (METEOSAT, MSG satellite) in the IR and WV channels and lightning data.

We will use GCD (Global Convective Diagnostic) algorithm developed at Aviation Weather Centre (AWC) of NOAA (National Oceanic and Atmospheric Administration). This algorithm is based on the idea that a deep convective cloud will not have any significant moisture above it.

This technique works quite well at identifying active deep convection and can be applied to all the world's geostationary satellites. However it does not always agree with lightning sensors. Low topped convection with lightning will be missed. We will extend the capabilities of GCD using lightning data. The new product will be validate over different cases in the central Italy using the C-band polarimetric radar of ISAC-CNR (Institute of Atmospheric Sciences and Climate-of the Italian National Research Council) Rome.
\end{abstract}

\section{Introduction}

The convective parts of the thunderstorms are for many reasons the most difficult to forecast, to reproduce in mesoscale models and even the most difficult to identify.

However, these active convective areas, representing the most dangerous part of the storms, are the most important. Thunderstorm detection is required for a large number of applications ranging from global climate studies to meteorological purposes, from civil protection aims to aircraft operations.

Mosher (2001) has proposed using the difference between the 6.7 micron water vapor (WV) and the 11 micron IR channels to identify deep convection and to monitor global convection since these two channels are available on the geostationary weather satellites. This technique, herein called

Correspondence to: C. M. Medaglia

(c.medaglia@isac.cnr.it)
GCD, originally, has been developed in the AWC-NOAA for generating High Level Significant Weather forecasts covering $2 / 3$ of the globe which include areas of deep convective activity that will affect aircraft, however, in our aims, this algorithm will be used for meteorological issues.

\section{Algorithm physical basis}

The physical concept behind the GCD algorithm is that thunderstorms lift WV and cloud particles to the top of the troposphere. Where there is active uplift to the top of the troposphere, the ir and wv channels of geostationary satellite sensors will have the same temperature. The wind at the thunderstorm top will transport the cloud ice particles and WV down wind. As the cloud ice particles advect away from the thunderstorm, they will gradually fall because of their mass. WV will also advect away from the thunderstorm, but will not fall. hence in the cirrus clouds down wind of the thunderstorm, there should be a slight temperature difference between the IR temperature of the cloud particles and the WV channel temperature of the WV. The current algorithm eliminates areas where the IR channel is at least $1^{\circ} \mathrm{C}$ warmer than the WV channel (Mosher, 2001).

First the algorithm was modified to take the differences between the channels in the original satellite image projections, and then to remap and composite the differences into a global composite (Martin et al., 2005).

\section{Preliminary results over Mediterranean Region}

The GCD algorithm has been applied over italian peninsula. We noted (Mosher, personal communications) that over italy the method is not working as well as it was doing over us territory. GCD algorithm shows some problem over italy and in particular over complex orography region. So that the main aims of this work are the understanding of the algorithm performances over italy and then the improvement of its capability. 
Table 1. Statistical results for different thresholds of differences between $T_{I R}$ and $T_{W V}$ (5 August 2004 at 16:30 UTC). POD (Probability of Detection) [y (yes-yes: pixels in which both GCD and radar detects convection); $\mathrm{n}$ (both GCD and radar don't consider pixels as convective)]; FAR (False Alarm ratio, it gives the percentage of the pixels in which there is no convection for radar and instead GCD detected convection), CSI (Critical Success Index ).

\begin{tabular}{lrrrrrrr}
\hline Thresholds (K) & $\mathbf{1}$ & $\mathbf{0 , 5}$ & $\mathbf{0 , 2 5}$ & $\mathbf{0}$ & $\mathbf{- 0 , 2 5}$ & $\mathbf{- 0 , 5}$ & $\mathbf{- 1}$ \\
Tot number pixels & 1641 & 1641 & 1641 & 1641 & 1641 & 1641 & 1641 \\
PODy & 24 & 26 & 27 & 29 & 28 & 20 & 14 \\
PODn & 90 & 90 & 89 & 88 & 88 & 87 & 87 \\
FAR & 75 & 73 & 72 & 70 & 71 & 79 & 85 \\
CSI & $\mathbf{1 8}$ & $\mathbf{1 7}$ & $\mathbf{1 6}$ & $\mathbf{1 2}$ & $\mathbf{8}$ & $\mathbf{4}$ & $\mathbf{1}$ \\
\hline
\end{tabular}

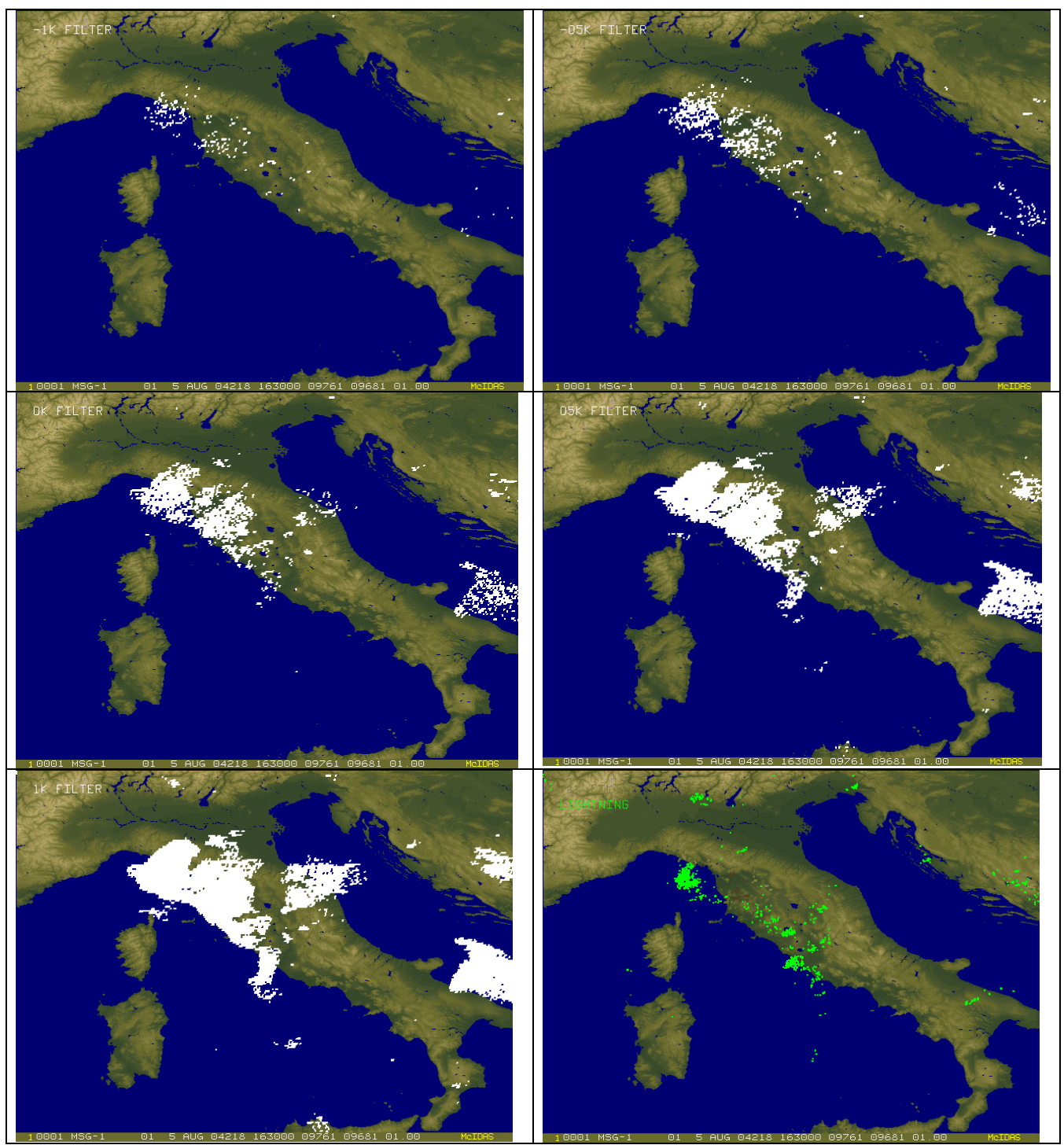

Fig. 1. Different thresholds applied to GCD algorithm over Italy for 5 August 2004 16:30 UTC. Left Upper panel is -1 K, left is -0.5 , in the middle line: the left panel represents $0 \mathrm{~K}$ threshold. Left lower panel is the $1 \mathrm{~K}$ threshold and the right panel shows lightning occurrence (green points) in the same window time of the MSG measurements. Lightning data have been measured by ZEUS network and kindly provided by NOA (National Observatory of Athens, Greece). 
To focus on this topic, the thunderstorm occurred over roman area on the 5 and 6 August 2004 has been studied. the available data for this case are:

- MSG measurements,

- Lightning data by Zeus network,

- C-band radar reflectivity measurements acquired by the ISAC-CNR Roma radar as validation dataset.

Figure 1 shows all the different pixels in which the algorithm recognizes the convection as a result of the differences between brightness temperatures in the MSG IR ( $\left.\mathrm{T}_{\mathrm{IR}}\right)$ and WV ( $\left.T_{\mathrm{WV}}\right)$ channels. The different maps are referred to values of the $T_{\text {ir }}-T_{W V}$ are respectively $-1,-0.5,0,0.25,0.5$, $1 \mathrm{~K}$.

Radar data are considered ground true. In particular we have chosen VMI (Vertical maximum Intensity of Reflectivity) parameter for individuating convective cells. Because the definition of convection is not so well established, we adopted $35 \mathrm{DBz}$ bottom threshold for considering the radar pixel a convective one. In order to validate the gcd performances we estimated the agreement between gcd estimations and radar measurements via statistical success index. We have performed for the area covered by the ISAC-CNR radar that cover the $250 \times 250 \mathrm{~km}$ region around Rome.

The statistical analysis for the comparison between GCD and radar (Table 1) shows that the Critical Index of Success (CSI) is around $18 \%$ for the highest threshold and goes down with the threshold value. Probability of detection for the noevent (PODn) is always very high, the detection convective pixels (PODy) is roughly between 20 and $29 \%$, and the false alarm ratio (FAR) is from 70 up to $85 \%$. Considering the positive thresholds, we reach always a CSI index greater than 10 . This results can be compared with the results obtained by Martin et al. (2005) validating GCD over pacific and atlantic oceans using goes data in many different meteorological situations. They used as validation the data measured by the Precipitation Radar (PR) aboard of TRMM (Tropical Rainfall Measurements Mission) satellite.

They found an agreement between GCD and radar lower than the one showed here (except for one case), even if it was better than the performances obtained considering the benchmark index ( $\mathrm{T}_{\mathrm{IF}}$ lower than $\left.215 \mathrm{~K}\right)$. Note that the study of Martin et al. (2005) considered convective a PR pixel, where the height of the storm was greater than $10 \mathrm{~km}$, the reflectivity was greater than $17 \mathrm{DBz}$. This condition appears stronger than the one we choose (VMI $>35 \mathrm{DBz}$ ), however, for geographical reason, the kind of storms considered are pretty different.

For this analysis, the original method has been applied to many radar volumes at any given $15 \mathrm{~min}$, between 16:00 and 18:00 UTC for the selected study. The results are similar to the showed case but more robust. The next step is the improvement of the algorithm for our issues and lightning measurements appeared like a good candidate to do that. The strongly relationship between lightning and convection

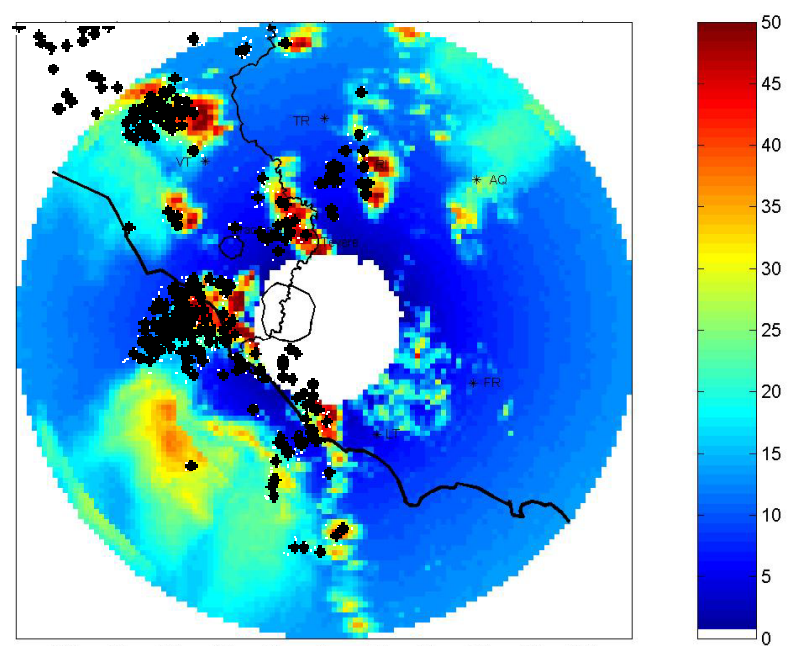

Fig. 2. 5 August 2004, 16:30 UTC: correspondence between radar map of Vertical Maximum Intensity (VMI) of Reflectivity and presence of lightning - represented by black asterisks - (contemporaneously within 10 min time window).

is well known and established (Peterson and Rutledge, 2001; Williams et al., 1992, Carey and Rutledge, 2000) and lightning data are continuously monitored over ocean and over land as well.

Figure 2 shows the temporal coincidence between lightning data and radar reflectivity for the selected case study. We developed a tool to insert lightning data (showed in Fig. 1 last panel) into the main GCD algorithm, considering the presence of lightning in a pixel like an addition information to GCD for the convection detection. The original radar spatial resolution $(2 \times 2 \mathrm{~km})$ has been degraded to $6 \times 6 \mathrm{~km}$ resolution in order to be comparable with lightning data. The same treatment has been done on the GCD output pixels. The new algorithm, considers convective all the pixel in which GCD detects convection using the 1 and 0 thresholds plus the lightning presence.

The results improve respect to the simple gcd and also with respect to the use of lightning data by themselves. The results are largely commented in the extension of this proceeding that is going to be published, but we anticipate that the CSI improves from the $12 \%$ or $18 \%$ (see Table 1 for GCD thresholds of $0 \mathrm{~K}$ or $1 \mathrm{~K}$ respectively) up to the $25 \%$.

\section{Conclusions and future work}

After this analysis over Italian Peninsula, we know the performances of GCD algorithm applied to MSG data. Comparing results obtained from the use of GCD using MSG data with to the performances of GCD using GOES data (come up close to the results obtained by Martin et al. (2005)), we obtained higher agreement with the radar data, even if we have to remember that Martin et al, used another "definition" of convection as true. However, also comparing GCD using MSG with GCD from Meteosat data, there is an 
improvements of the performances, as we found previously (not showed).

As future work, we will improve the quality of the information obtained by lightning to better detect thunderstorm convection. In particular we are conducting two studies: one focuses on using lightning flash rate instead of lightning presence, the other focuses on using a temporal dynamic approach for both infrared and lightning data.

Acknowledgements. The Italian team has been funded by the Italian National Group for Prevention from Hydro-Geological Disasters (GNDCI). The authors wish to thank E. Defer (NOANational Observatory of Athens) for providing lightning data; D. Martin and R. Kohrs (SSEC - Space Science and Engineering Center, Madison, WI) for their precious inputs and the useful conversations on this topic; F. Mosher (Embry-Riddle Aeronautical University, Daytona Beach, FL) for given us GCD algorithm. Finally we thank J. Weinman (Univ. of Washington, Seattle, WA) for his initial advances and help in this work.

Edited by: L. Ferraris

Reviewed by: anonymous referees

\section{References}

Carey, L. D. and Rutledge, S. A.: On the Relationship Between Precipitation and Lightning in Tropical Island Convection: A Cband Polarimetric Radar Study, Monthly Weather Review, 128, 2687-2710, 2000.

Martin, D. W., Mosher, F. R., Kohrs, R. A.: Over Ocean validation of the global convective diagnostic, in: Preprints, 13th Conference on Satellite Meteorology and Oceanography (20-24 September 2004; Norfolk, VI), in press, 2005.

Mosher, F. R.: A satellite diagnostic of global convection, in: Preprints, 11th Conference on Satellite Meteorology and Oceanography (15-19 October 2001; Madison, WI), 416-419, 2001.

Peterson, W. A. and Rutledge, S. A.: Regional Variability in Tropical Convection: Observations from TRMM, J. Climate, 14, 3566-3585, 2001.

Williams, E. R., Rutledge, S. A., Geotis, S. C., Renno, N., Rasmussen, E., and Rickenbach, T.: A radar and electrical study of tropical hot towers, J. Atmos. Sci., 49, 1386-1395, 1992. 